\title{
Bilateral Hypoglossal Palsies: A Late Complication of Curative Radiotherapy
}

\author{
Eamon F. Johnston, Alex J. Hammond and J. Gregory Cairncross
}

\begin{abstract}
A 57 year old man developed bilateral hypoglossal nerve palsies 6 years after radiotherapy for carcinoma of the uvula. Follow-up over 2 years has demonstrated no evidence of tumour recurrence and no sign of neurological improvement. Reactive fibrosis and vascular insufficiency secondary to radiation and may have lead to hypoglossal nerve compression and infarction.
\end{abstract}

RÉSUMÉ: Paralysie bilatérale de l'hypoglosse: une complication tardive de la radiothérapie curative Un patient âgé de 57 ans a développé une paralysie bilatérale de l'hypoglosse 6 ans après avoir subi de la radiothérapie pour un carcinome de la luette. Le suivti sur une période de 2 ans n'a pas mis en évidence de récidive de la tumeur ou de signe d'amélioration neurologique. La fibrose réactionnelle et l'insufficance vasculaire secondaires à la radiothérapie peuvent avoir entraîné une compression et un infarcissement de l'hypoglosse.

Can. J. Neurol. Sci. 1989; 16:198-199

The list of neurological disorders caused by or related to therapeutic radiation includes the "somnolence syndrome", cerebral atrophy with dementia, cerebral necrosis, Lhermitte's symptom, necrotic myelopathy, brachial plexopathy and cauda equina/lumbosacral plexopathy. Peripheral nerves, including the cranial nerves, are remarkably resistant to radiation injury. Unilateral hypoglossal palsy is a recognized complication of radiation treatment for head and neck cancer. ${ }^{1,2}$ We now report an unusual case of bilateral hypoglossal neuropathy following radiotherapy for carcinoma of the uvula.

\section{Case Report}

In April 1986, a 63 year old man with diabetes mellitus and a history of uvular carcinoma woke one morning with pain at the angle of the mandible radiating to the left occiput and garbled speech. He noted difficulty manipulating food in his mouth and excessive salivation. He recalled intermittent difficulty with speech and mastication over the preceding four months. Careful examination of the mouth and oropharynx revealed no evidence of recurrent tumour. The submandibular soft tissues were thickened. There was no evidence of cervical lymphadenopathy. The neurological examination revealed bilateral hypoglossal nerve palsies with marked wasting and weakness of the tongue (figure 1). There were no other cranial nerve abnormalities. A mild sensory polyneuropathy with absent ankle reflexes was noted. Blood sugars were moderately well controlled with diet and chlorpropamide $(250 \mathrm{mg} / \mathrm{day})$. A CT scan of the base of skull and extracranial soft tissues revealed no evidence of bone erosion or mass suggest- ing tumour recurrence. Linear streaking and loss of soft tissue planes were noted medial to the angle of the mandible on the right. There has been no clinical change over 2 years. A recent MRI scan revealed fatty atrophy of the tongue with no areas of abnormal signal in the medulla or along the course of the hypoglossal nerves.

In 1980, this former smoker and moderately heavy drinker presented with a 2 month history of sore throat and minor bleeding from the mouth. Examination revealed a $2.5 \mathrm{~cm}$ ulcerating lesion replacing the

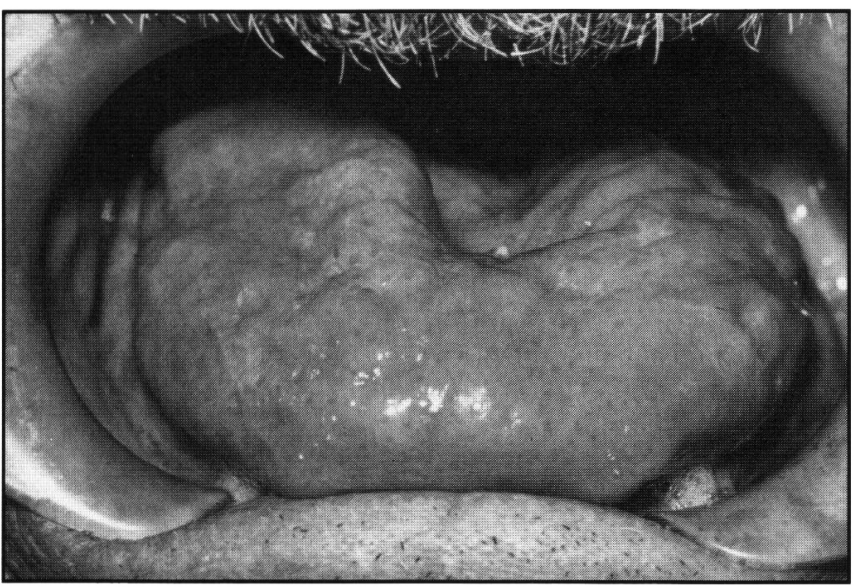

Figure I - Photograph demonstrating bilateral atrophy of the tongue.

From the Departments of Oncology and Clinical Neurological Sciences, University of Western Ontario and London Regional Cancer Centre, London

Received June 22, 1988. Accepted September 16, 1988

Reprint requests to: J. Gregory Cairncross, MD, London Regional Cancer Centre, 790 Commissioners Road East, London, Ontario, Canada N6A 4L6 
uvula and extending onto the soft palate. There were no lymph nodes palpable in the neck and the remainder of the physical examination was normal. Excisional biopsy demonstrated a moderately well differentiated squamous cell carcinoma. He was treated with external beam irradiation using a 780 Theratron cobalt unit. He received $6100 \mathrm{cGy}$ delivered in 25 fractions over 5 weeks using equally weighted parallel opposed fields to a $9 \times 7 \mathrm{~cm}$ portal as shown in figure 2 . He also received $5000 \mathrm{cGy}$ in 25 fractions over 5 weeks to an adjoining single anterior neck field. Three weeks after completing treatment there was no evidence of residual tumour and apart from a dry mouth he was asymptomatic. Five months later he experienced sudden pain in the left cheek which woke him from sleep. Subsequently he complained of aching discomfort in both cheeks which developed insidiously and persisted for 6 months. Articulation and mastication were normal. The pain subsided spontaneously and he remained well until his neurological difficulties began 5 years later.

\section{DISCUSSION}

This patient developed permanent, severely disabling bilateral hypoglossal nerve palsies 66 months after radiation treatment for carcinoma of the uvula. The hypoglossal neuropathies appear to be radiation-induced. The distinction between tumour recurrence and radiation-induced cranial nerve palsy is critical. The physical findings, imaging studies and observation over time support the latter. To our knowledge there are no previous descriptions of isolated bilateral hypoglossal nerve injury following radiation.

In clinical practice radiation-induced cranial nerve injury is distinctly uncommon. Cranial neuropathy in the cancer patient is far more likely to be caused by leptomeningeal or base of skull metastases, than radiation. Nevertheless, there are now a small number of well documented cases in which cranial nerve injury has complicated curative radiotherapy. The hypoglossal nerve appears to be the most commonly affected. Cheng and

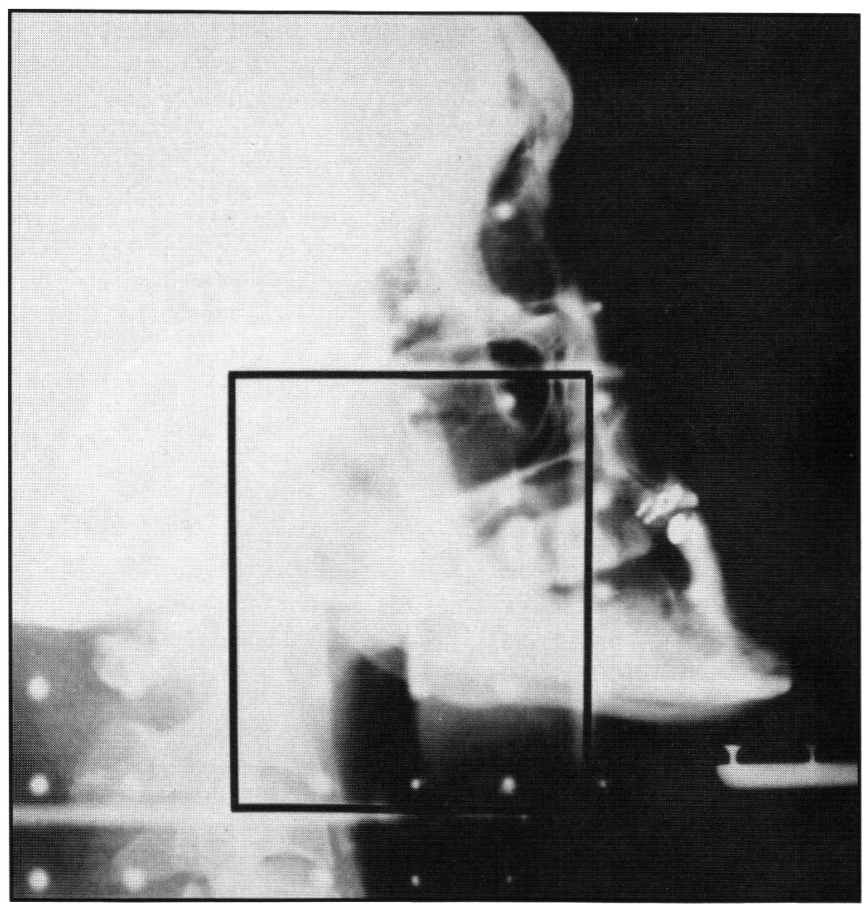

Figure 2 - Lateral radiograph of the skull and upper cervical spine with the irradiated field outlined.
Schultz reported four cases of unilateral hypoglossal nerve palsy with hemi-atrophy of the tongue developing 3-9 years after radiation treatment for head and neck cancer. ${ }^{1}$ Neck fibrosis preceded hypoglossal palsy in all instances. Berger and Bataini2 reported 35 instances of cranial neuropathy in 25 patients following radical radiotherapy for head and neck tumours. The hypoglossal nerve was involved in 19 cases, the vagus in 9 and the spinal-accessory in 5. Unilateral hypoglossal palsy was the sole cranial nerve abnormality in 16 of 25 patients. The cranial nerve palsies were noted 12-145 months after radiation treatment with a latency period correlating inversely with the total treatment dose. Radiation injury of the optic, 2.3.4 trigeminal $^{2}$ and recurrent laryngeal branch of the vagus ${ }^{5,6}$ have also been reported. Descriptions of abducens and auditory nerve toxicity are less convincing.

The mechanism by which radiation damages cranial nerves is unknown. This patient was treated to $6100 \mathrm{cGy}$ with large fractional doses (244 cGy). A direct, dose-dependent toxic effect of ionizing radiation on neural tissue cannot be excluded. However, the physical examination and CT scan findings in this case suggest a significant submandibular soft tissue fibrotic reaction to radiation. Nerve infiltration and compression occurs in areas of intense radiation fibrosis (e.g. radiation brachial plexopathy). Direct nerve injury, nerve ischemia, or a combination of the two may result. It is tempting to speculate that underlying microvascular disease secondary to diabetes mellitus predisposed this patient to ischemic hypoglossal nerve injury as the fibrosis intensified.

The hypoglossal nerve seems to be more susceptible to radiation injury than are other cranial nerves in the region (c.g. vagus, spinal accessory). There is no satisfactory explanation for this apparently unique vulnerability.

The clinical importance of radiation-induced bilateral hypoglossal nerve palsies is underscored by the seriousness of the disability that ensues. Whereas unilateral hypoglossal neuropathies from whatever cause are usually asymptomatic, bilateral palsies severely impair speech, chewing and swallowing. Our patient has badly garbled speech and eats only pureed foods. There has been no improvement over two years and we have every reason to believe that the neurological disability is permanent.

\section{REFERENCES}

I. Cheng VST, Schulz MD. Unilateral hypoglossal nerve atrophy as a late complication of radiation therapy of head and neck carcinoma: a report of four cases and a review of the literature on peripheral and cranial nerve damages after radiation therapy. Cancer 1975; 35: 1537-1544.

2. Berger PS, Battaini JP. Radiation-induced cranial nerve palsy. Cancer 1977; 40: 152-155.

3. Schukovsky LJ, Fletcher GH. Retinal and optic nerve complications in a high dose radiation technique of ethmoid sinus and nasal cavity. Radiology 1972; 104: 629-634.

4. Ross HS, Rosenberg S, Friedman AH. Delayed radiation necrosis of the optic nerve. Am J Ophthal 1973; 76: 683-686.

5. Craswell PWT. Vocal cord paresis following radioactive iodine therapy. Br J Clin Pract 1972; 26: 571-572.

6. Westbrook KC, Ballantyne AJ, Eckler NE, et al. Breast cancer and vocal cord paralysis. South Med J 1974; 67: 805-807. 